\title{
The piperazine compound ASP activates an auxin response in Arabidopsis thaliana
}

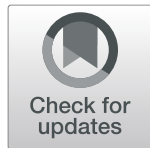

Fengyang Xu, Shuqi Xue, Limeng Deng, Sufen Zhang, Yaxuan Li and Xin Zhao*

\begin{abstract}
Background: Auxins play key roles in the phytohormone network. Early auxin response genes in the AUX/ IAA, SAUR, and GH3 families show functional redundancy, which makes it very difficult to study the functions of individual genes based on gene knockout analysis or transgenic technology. As an alternative, chemical genetics provides a powerful approach that can be used to address questions relating to plant hormones.

Results: By screening a small-molecule chemical library of compounds that can induce abnormal seedling and vein development, we identified and characterized a piperazine compound 1-[(4-bromophenoxy) acetyl]-4-[(4-fluorophenyl) sulfonyl] piperazine (ASP). The Arabidopsis DR5::GFP line was used to assess if the effects mentioned were correlated with the auxin response, and we accordingly verified that ASP altered the auxin-related pathway. Subsequently, we examined the regulatory roles of ASP in hypocotyl and root development, auxin distribution, and changes in gene expression. Following ASP treatment, we detected hypocotyl elongation concomitant with enhanced cell elongation. Furthermore, seedlings showed retarded primary root growth, reduced gravitropism and increased root hair development. These phenotypes were associated with an increased induction of DR5::GUS expression in the root/stem transition zone and root tips. Auxin-related mutants including tir 1-1, aux $1-7$ and axr2-1 showed phenotypes with different rootdevelopment pattern from that of the wild type (Col-0), and were insensitive to ASP. Confocal images of propidium iodide (PI)-stained root tip cells showed no detectable damage by ASP. Furthermore, RT-qPCR analyses of two other genes, namely, Ethylene Response Factor (ERF115) and Mediator 18 (MED18), which are related to cell regeneration and damage, indicated that the ASP inhibitory effect on root growth was not attributable to toxicity. RT-qPCR analysis provided further evidence that ASP induced the expression of early auxin-response-related genes.
\end{abstract}

Conclusions: ASP altered the auxin response pathway and regulated Arabidopsis growth and development. These results provide a basis for dissecting specific molecular components involved in auxin-regulated developmental processes and offer new opportunities to discover novel molecular players involved in the auxin response.

Keywords: Chemical genetics, Auxin response, ASP, Phytohormone, Auxin signaling

\footnotetext{
*Correspondence: zhaox0521@126.com

Fengyang Xu and Shuqi Xue are co-first authors

College of Life Sciences, Capital Normal University, Beijing 100048, China
}

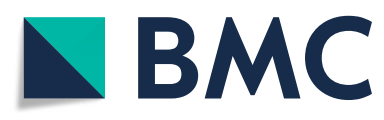

(c) The Author(s). 2020 Open Access This article is licensed under a Creative Commons Attribution 4.0 International License, which permits use, sharing, adaptation, distribution and reproduction in any medium or format, as long as you give appropriate credit to the original author(s) and the source, provide a link to the Creative Commons licence, and indicate if changes were made. The images or other third party material in this article are included in the article's Creative Commons licence, unless indicated otherwise in a credit line to the material. If material is not included in the article's Creative Commons licence and your intended use is not permitted by statutory regulation or exceeds the permitted use, you will need to obtain permission directly from the copyright holder. To view a copy of this licence, visit http://creativecommons.org/licenses/by/4.0/ The Creative Commons Public Domain Dedication waiver (http://creativecommons.org/publicdomain/zero/1.0/) applies to the data made available in this article, unless otherwise stated in a credit line to the data. 


\section{Background}

Auxins are a class of phytohormones that regulate almost every aspect of plant growth and development, including root development, vascular tissue differentiation and tropisms [1, 2]. Indole-3-acetic acid is the best known auxin, and its receptors and signaling pathway are well known [3]. The core components of this pathway are the F-box-containing TRANSPORT INHIBITOR RESISTANT1/AUXIN SIGNALING F-BOX (TIR1/AFB) proteins, the transcriptional corepressors AUXIN/INDOLE-3-ACETIC ACID (Aux/IAA), and the AUXIN RESPONSE FACTOR (ARF) transcription factors. Auxin binds to the receptor TIR1 and members of the AFB family, which are the substrate-recognition subunit of the SUPPRESSOR OF KINETOCHORE PROTEIN 1 (SKP1)/CULLIN1/F-Box (SCF) E3 ubiquitin ligase complex to promote the interaction between TIR1/AFB and Aux/IAA by triggering polyubiquitination and degradation of Aux/IAA. The removal of Aux/IAA allows ARF to activate or repress early auxin-responsive gene transcription [4]. All of these elements contribute to auxin responses.

A growing body of evidence shows that auxin and other phytohormones-signaling pathways can interact with each other; thus, contributing to the complexity of auxin-mediated regulation of plant growth and development $[5,6]$. For example, the interaction between auxin and cytokinin pathways has been shown to be important for maintaining plant root and shoot meristematic activity [7]. In this case, hypocotyl cytokinin inhibits auxin and aspartate conjugation, thereby promoting an increase in the concentration of free auxin and, consequently, cytokinin oxidase activity, which in turn leads to cytokinin degradation. In addition, free auxin and auxin conjugates can also inhibit $\beta$-glucosidase activity and cause a reduction or loss of cytokinin activity [8]. Considerable attention has also focused on the crosstalk between ethylene and auxin signaling pathways, and it has been unequivocally demonstrated that auxin and ethylene regulate the formation of the apical hook, root, and hypocotyl growth. In Arabidopsis, the ethylene mutant ctr 1 showed a short primary root due to inhibited cell division and elongation. This phenotype was associated with an increase in the expression of the auxin transporter PIN2 and a stronger DR5::GFP response [9]. Consistently, in cotton, the effect of ethylene on leaves has been found to be related to a reduction of auxin polar transport [10]. Moreover, auxin can induce ethylene biosynthesis by regulating the expression of 1aminocyclopropane-1-carboxylic acid (ACC) synthase, a key enzyme in ethylene biosynthesis. In $A$. thaliana, the promoter region of ACS4 encoding ACC synthase, which contains an auxin response element, can be induced by auxin. In plants, guard cells open and close stomata to control transpiration and regulate gas exchange in leaves. Both cytokinins and auxins have been demonstrated to regulate stomatal behavior [11]. Furthermore, auxin and abscisic acid (ABA) play antagonistic roles in regulating stomatal opening and closure. Such antagonism is achieved by a precise regulation of the ion channel activity of the guard cells, which can reduce IAA or increase $\mathrm{ABA}$, and regulate cytoplasmic $\mathrm{pH}$, thereby affecting the expansion of guard cells [12].

Chemical genetics has been widely used to study biological systems because some small-molecule compounds can specifically bind proteins to interfere with developmental processes $[13,14]$. The application of chemical genetics in plants has allowed great progress in identifying plant hormone receptors [15], and signal transduction and plant genetic diversity studies [16].

As important signaling molecules, auxins play a major role in plant growth and development. However, study of the auxin response pathway has been limited to a certain extent by the functional redundancy of early response genes, whereas mutations in key genes can cause plant infertility or lethality. In this study, we examined the effects a small-molecule piperazine compound, ASP, on $A$. thaliana phenotypes, with the aim of identifying and characterizing specific molecular components involved in auxin-regulated developmental processes. Our findings highlight the advantages of using a chemical genetics approach to address questions relating to phytohormone signaling pathways.

\section{Results}

Screening of small-molecule compounds that alter leafvein patterns and the auxin response in A. thaliana

In an attempt to identify small-molecule compounds that can affect leaf vein patterns, we screened a smallmolecule compound library containing 3800 compounds, using 10-day-old seedlings of $A$. thaliana line Q0990. The expression of a vascular precursor cell reporter in Q0990 seedlings enabled us to screen for chemically induced defects in vascular development [17]. Seeds of Q0990 were grown in 96-well plates, in which each well contained a $10 \mu \mathrm{M}$ solution of different compounds. Among these compounds, we selected 11 small molecules having an effect on vein pattern and leaf shape, all of which promoted central-vein thickening and an increase in the number of parallel vascular bundles (Fig. 1a, b).

To determine whether these physiological effects were correlated with a molecular auxin response, we employed transgenic Arabidopsis seedlings in which the synthetic auxin response element DR5 was fused to the green fluorescent protein (GFP) reporter. Among the 11 initially selected chemicals, we identified six candidates by confocal microscopy, in which the expression level of 

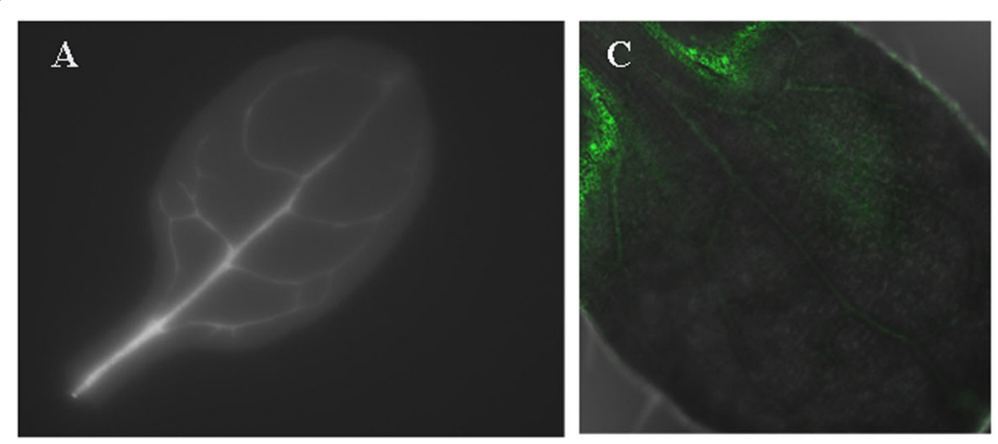<smiles>O=C(COc1ccc(Br)cc1)N1CCN(S(=O)(=O)c2ccc(F)cc2)CC1</smiles>
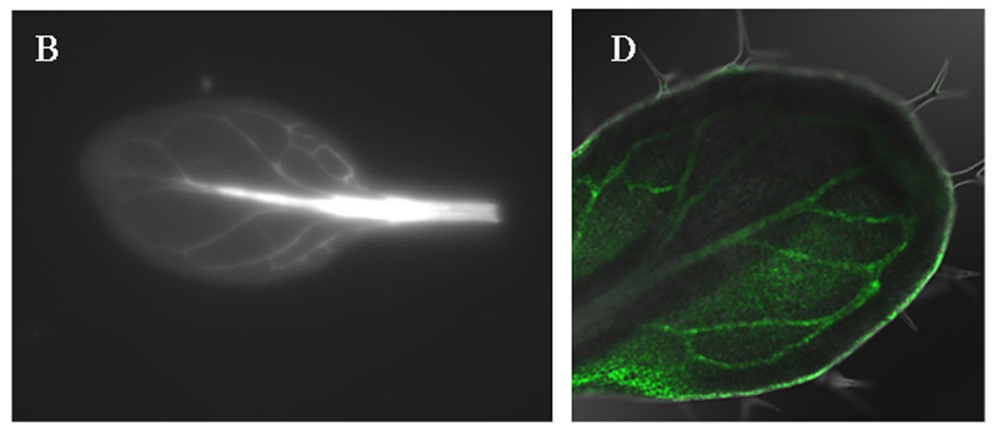<smiles>O=C(O)Cc1c[nH]c2ccccc12</smiles>

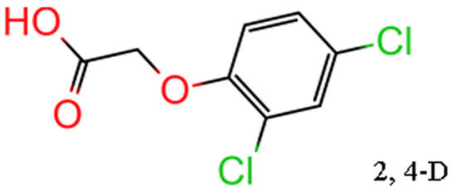

Fig. 1 Chemical genetics screening identified a novel compound ASP that produced abnormal leaf-vein pattern. a-b: Leaf vein pattern of 10-dayold seedlings of Q0990 line grown on 1/2 MS medium (a) and 1/2 MS medium with $10 \mu \mathrm{M}$ ASP (b). c-d: Observation of 10-day-old seedlings of DR5::GFP reporter line grown on 1/2 MS medium (c) and 1/2 MS medium with $10 \mu \mathrm{M}$ ASP (d) by confocal scanning laser microscope. E: Chemical structural formula of endogenous auxin indole-3-acetic acid (IAA), synthetic auxin 2,4-dichlorophenoxyacetic acid (2,4-D), and small-molecule compound 1-[(4-bromophenoxy) acetyl]-4-[(4-fluorophenyl) sulfonyl] piperazine (ASP)

the reporter gene increased significantly after treatment, and the distribution of which was consistent with the location of leaf vein formation (Fig. 1c, d). Here, we focused on analyzing the character of the piperazine compound 1-[(4-bromophenoxy) acetyl]-4-[(4-fluorophenyl) sulfonyl] piperazine (ASP) and used it as a chemical tool to study the auxin signaling pathway.

Notably, we found that ASP showed structural similarities to the synthetic auxin 2,4-dichlorophenoxyacetic acid (2,4-D) and endogenous IAA, both of which contain an unsaturated aromatic ring and a carboxylic acid side chain (Fig. 1e). This structural similarity reminded us of the functional similarity.

\section{ASP can inhibit the elongation of primary and lateral roots, promote root hair development, and reduce gravitropism in A. thaliana}

Phytohormones such as auxin and ethylene play crucial roles in the regulation of root growth [18]. To further characterize ASP activity, we accordingly performed a series of root assays. Following treatment of 6-day-old seedlings of wild-type $A$. thaliana (Col-0) with different concentrations of ASP and 2,4-D, we found that both compounds had clear concentration-dependent inhibitory effects on root growth (Fig. 2a, b) (Additional file 1: Fig. S1). When we measured primary root length at 24-h intervals, we found that the rate of primary root cumulative growth was reduced in the $5 \mu \mathrm{M}$ ASP and $30 \mathrm{nM}$ 2,4-D treatments (Fig. 2c). Furthermore, we observed that $30 \mathrm{nM}$ 2,4-D and $4 \mu \mathrm{M}$ ASP had similar effects on primary root growth (Fig. 2a, b) (Additional file 1: Fig. S1), and that under these treatments, the inhibition rate of primary root growth was 50\%. Therefore, in subsequent experiments, $30 \mathrm{nM}$ 2,4-D was used as positive control treatment.

Then we observed the lateral root growth in 9-day-old seedlings, we found that $10 \mathrm{nM} 2,4-\mathrm{D}$ increased the lateral root density, however, $30 \mathrm{nM}$ 2,4-D inhibited such roots formation (Fig. 2d). When seedlings were treated with $2 \mu \mathrm{M}$ and $5 \mu \mathrm{M}$ ASP, we observed the emergence of numerous lateral roots on the primary root, although lateral root elongation was inhibited (Additional file 2: Fig. S2). Given that the effect of $10 \mathrm{nM} 2,4-\mathrm{D}$ and $2 \mu \mathrm{M}$ ASP had similar effects on the lateral root density of WT, we speculated that although ASP promoted lateral root initiation, it inhibited lateral root elongation.

Gravitropism is the process whereby plants orientate their root growth toward gravity, and is a necessary response that ensures roots grow down through the soil [19]. We examined whether ASP had an effect on the gravitropic response of Col-0 seedlings by rotating seedlings grown vertically on $2 \mu \mathrm{M}$ ASP-containing medium, and accordingly found that ASP retarded the rate of the gravitropic response. Thus, at $12 \mathrm{~h}$ after treatment, 
A
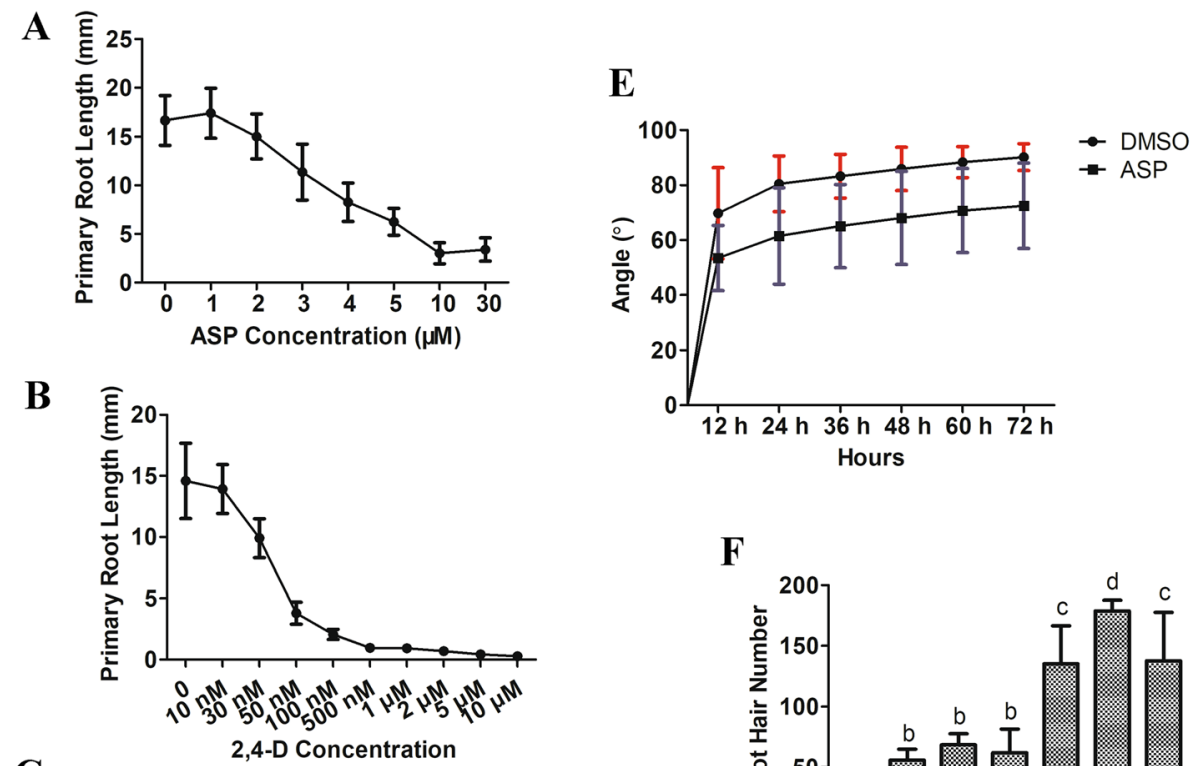

C

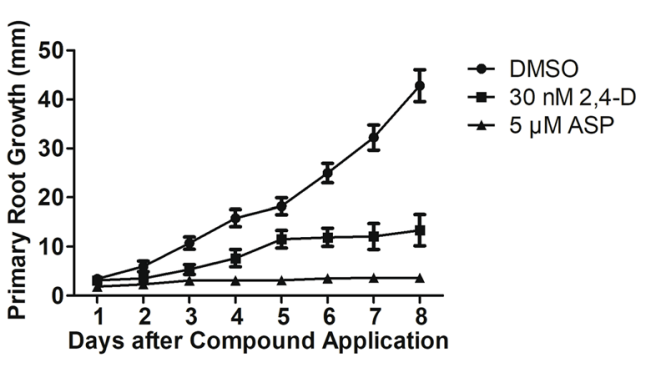

$\mathbf{F}$

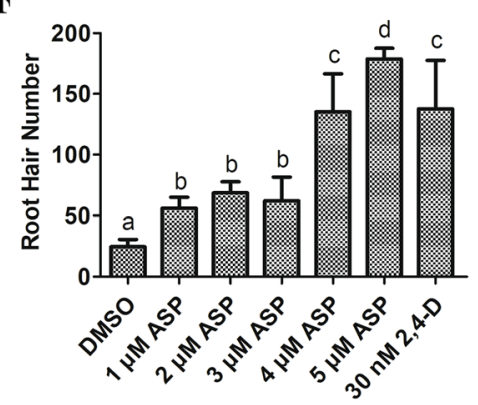

$\mathbf{G}$
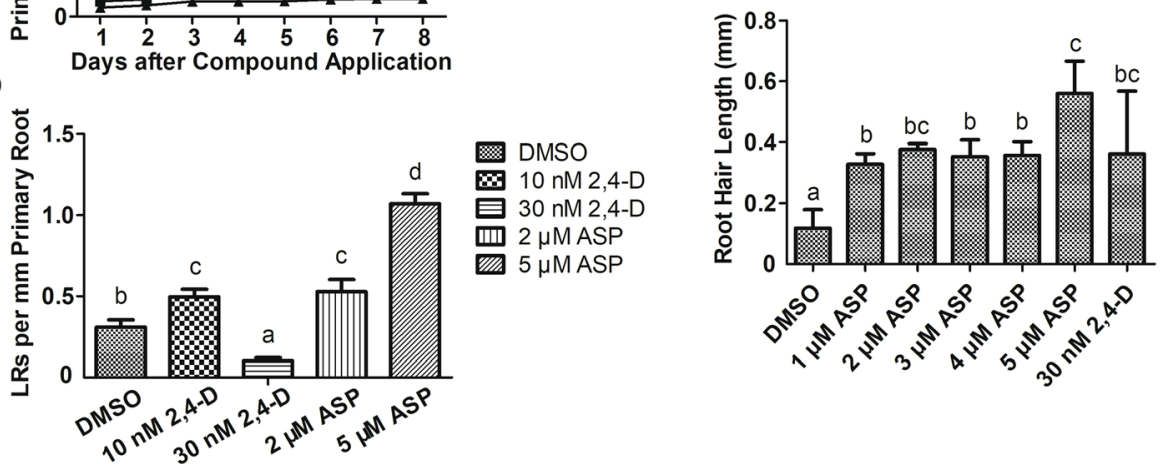

Fig. 2 ASP decreased root growth in A. thaliana. a: Dose-response curve for wild type (Col-0) seedlings on ASP. Seedlings were treated for six days with different concentrations of ASP from 0 to $30 \mu \mathrm{M}$ as indicated on X axis. b: Dose-response curve for Col-0 seedlings on 2,4-D. The concentrations of 2,4-D were from $10 \mathrm{nM}$ to $10 \mu \mathrm{M}$ as indicated on $\mathrm{X}$ axis. c: The cumulative rate of primary root length measured at 24-h interval. Seedlings were treated with $5 \mu \mathrm{M}$ ASP and $30 \mathrm{nM}$ 2,4-D respectively. DMSO was used as control. Each point represented the mean of at least 20 measurements. Error bars indicated the standard deviation. d: Effect of ASP and 2,4-D on lateral root density of WT seedlings. Seedlings were grown on 1/2 MS medium supplemented with $10 \mathrm{nM}$ 2,4-D, $30 \mathrm{nM}$ 2,4-D, $2 \mu \mathrm{M}$ ASP and $5 \mu \mathrm{M}$ ASP for nine days. DMSO was used as control (number of samples $N=10, P<0.05$ ). e: ASP reduced the gravitropic response. $2 \mu \mathrm{M}$ ASP was used for gravitropism test. Col-0 seedlings grown on agar medium were rotated $90^{\circ}$ at time 0 . The angle of curvature from the horizontal was measured at the times indicated. Each point represents the mean of 24 measurements. Error bars indicate the standard deviation. $\mathbf{f}$ and $\mathbf{g}$ : Root hair of Col-0 seedlings response to ASP and 2,4-D. Seedlings vertically grown on the medium supplemented with 0-5 $\mu \mathrm{M}$ ASP and $30 \mathrm{nM}$ 2,4-D for six days. Root hair upward root tips $5 \mathrm{~mm}$ was selected to measure and count (number of samples $N=50, P<0.05$ ). f: Statistics of root hair number. $\mathbf{g}$ : Measurements of root hair length

reorientation of the roots of ASP-treated plants showed noticeable inhibition, with a growth angle of $53^{\circ} \mathrm{com}-$ pared with the $70^{\circ}$ of control plant roots. This trend became increasing more pronounced with continued root growth, and when final measurements were taken at 72 $\mathrm{h}$ after treatment, we found that the primary roots of
ASP-treated plants had not bent to an angle of $90^{\circ}$ (Fig. 2e, Additional file 3: Fig. S3).

Root hair development has been shown to be regulated by multiple plant hormones [20], and in the present study, we observed an increase in the number of root hairs growing on Col- 0 seedlings in response to $1-5 \mu \mathrm{M}$ 
ASP treatment; further, the length of these root hairs changed significantly (Fig. 2f, g). Moreover, we observed that the growth of root hairs occurred closer to the root tips (Additional file 4: Fig. S4). These findings thus indicated that ASP can inhibit primary and lateral root growth, retard the gravitropic response, and promote root hair production and growth in WT Arabidopsis seedlings.

Based on the effect of ASP on root growth, we suspected that root inhibition was due to the toxicity of ASP. We tested this possibility by using propidium iodide (PI)-stained root tip cells of 5-day-old seedlings treated with different concentrations of ASP, IAA and 2, 4-D. PI is a nucleic-acid stain that can only penetrate cells with damaged or leaking cell membranes [21]. Confocal images revealed no detectable cellular damage due to treatment with ASP, IAA or 2,4-D, thereby indicating that the ASP-induced changes in root morphology are not associated with DNA damage or cell death (Additional file 5: Fig. S5).

\section{ASP promotes hypocotyl elongation in $A$. thaliana}

In general, well-known auxins (e.g., IAA, 2,4-D, 1-NAA) have no discernable effects on hypocotyl elongation [22]. In the present study, however, we found that treatment of $A$. thaliana with different ASP concentrations promoted hypocotyl elongation; furthermore, it had no clear concentration-dependent effects on 5-day-old seedlings (Fig. 3a, Additional file 1: Fig. S1). We observed semithin sections of hypocotyl tissue using light microscopy and measured cell length after 7 days of treatment with ASP to examine its effect on hypocotyl elongation at the cellular level. Longitudinal sections revealed that the hypocotyl cells of ASP-treated plants were significantly longer than those of control plants (Fig. 3b, Additional file 6: Fig. S6). Accordingly, these observations indicated that ASP enhanced hypocotyl growth by promoting cell elongation.
ASP has differing effects on auxin signaling mutants, and can induce the differential expression of several key genes in the auxin signaling pathway

To further explore whether ASP acted through the auxin signaling pathway, we assayed the phenotypes of three auxin signaling mutants, namely, tir $1-1$, axr2-1 and aux 1-7. Treatment with 1-5 $\mu \mathrm{M}$ ASP increased the hypocotyl length in tir1-1 and aux 1-7 relative to controls; and all three mutants showed no obvious root growth reduction. Furthermore, the promotion effects on the number and length of root hair were weakened in these mutants (Additional file 7: Fig. S7, Additional file 8: Fig. S8). In treatment with $4 \mu \mathrm{M}$ ASP that inhibited root elongation rate by $50 \%$, hypocotyl length of WT seedlings actually increased, with the promotion effect in tirl-1 and aux $1-7$ mutants being similar or larger than in the WT (Fig. 4a). However, compared with WT, the inhibitory effect in primary root growth was not significant in tir1-1, axr2-1 and aux 1-7 (Fig. 4b). Meanwhile, the root hair number and length in tir 1-1 and aux1-7 had no obvious changes by $4 \mu \mathrm{M}$ ASP treatment, indeed in axr2-1 they showed significant reduction (Fig. 4c, d). From these results we concluded that the sensitivity of tir $1-1$ and $a u x 1-7$ to ASP in the root was different from that in the hypocotyl; therefore, we hypothesized that ASP affected root and hypocotyl growth via different pathways.

In addition, we used seedling lines containing the auxin response element DR5 fused to the $\beta$ glucuronidase (GUS) reporter to examine whether the effects of ASP were correlated with the auxin response. Histochemical staining of DR5::GUS seedlings grown for $24 \mathrm{~h}$ in the presence of $5 \mu \mathrm{M}$ ASP revealed strong GUS expression in the cotyledons (Fig. 5a), the root/stem transition zone (Fig. 5b), and root tips (Fig. 5c). It is well known that auxin inhibits root growth and promotes root hair development [23]; thus, increased auxin accumulation implies root inhibition and root hair promotion.
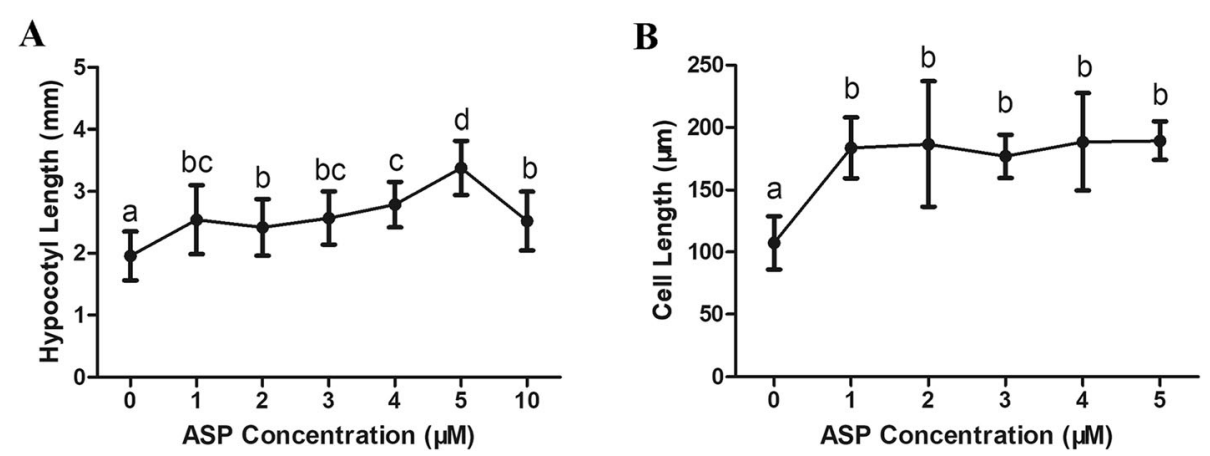

Fig. 3 ASP promoted hypocotyl elongation in Col-0 seedlings. a: Measurements of hypocotyl length in five-day-old seedlings under ASP treatment. The concentrations of ASP application were indicated on X axis. b: Statistical results of hypocotyl cell length. Hypocotyl cells were from semi-thin longitudinal section cut $(N=60, P<0.05)$ 


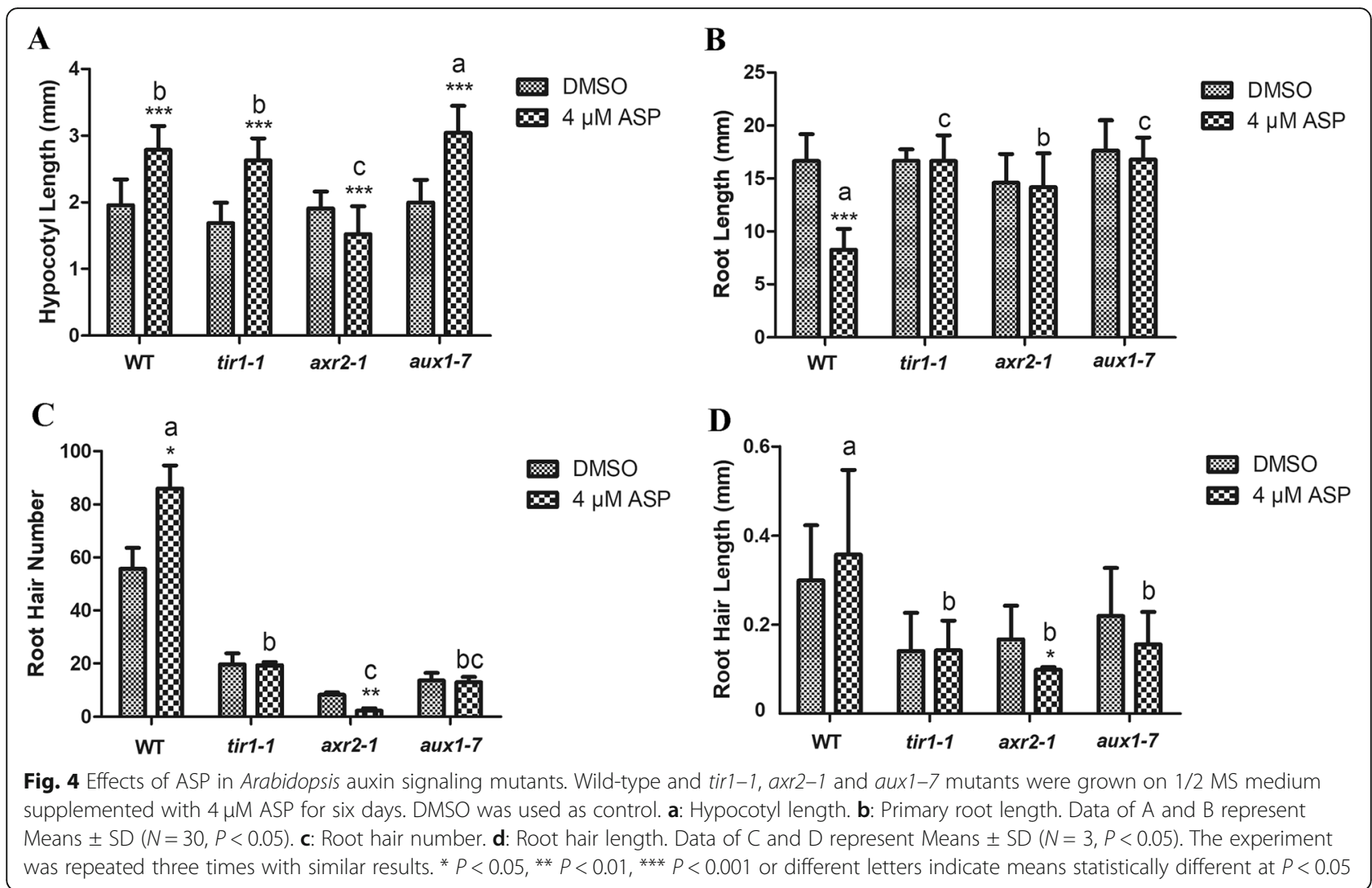

On the basis of the aforementioned results, we hypothesized that ASP might induce auxin-like responses, and accordingly sought to examine the ability of ASP to induce auxin-response genes in 6-day-old seedlings. To this end, we performed real-time quantitative PCR (RT-qPCR) to analyze the expression of early auxin-response genes including, $A u x / I A A, G H 3$ and SAUR within $2 \mathrm{~h}$ of treatment with ASP.

As expected, we found that ASP significantly upregulated the auxin-responsive genes in WT seedlings. IAA 2 expression increased at $15 \mathrm{~min}$ by IAA treatment and then decreased over the following $2 \mathrm{~h}$. However, with ASP treatment, the expression level increased at 1 and $2 \mathrm{~h}$ sampling-time points. The trend of the relative expression of the GH3.5 was generally consistent under IAA and ASP treatment. The increase in gene expression level was evident after $1 \mathrm{~h}$ treatment, and an over four-fold increase was observed after $2 \mathrm{~h}$ of ASP treatment. The response of SAUR23 to IAA and ASP was rapid. After $15 \mathrm{~min}$ of treatment, the expression level had increased significantly (Fig. 6). Although the expression patterns of these genes were not exactly unanimous, the altered expression levels of the examined genes indicated that ASP treatment triggered auxin activity.

\section{Discussion}

Auxins were the first class of plant hormones to be discovered. Auxins play an important role in plant morphogenesis and development, including shoot and root growth and hypocotyl elongation [24]. IAA is the main form of auxin in plants, although there are numerous other substances with auxin-like activity including, indole-3-butanoic acid (IBA), 4-chloroindole-3-acetic acid (4-Cl-IAA), and phenylacetic acid (PAA). These latter compounds have chemical structures similar to that of IAA and, accordingly, are considered to be plant endogenous auxins [25]. Along with the rapid development of synthetic chemical growth regulators, a number of auxin-like compounds have been identified and extensively applied in plant research [26].

Taking advantage of the power of chemical genetics, we performed a small-molecule library screening and obtained six candidates that were considered to be potentially relevant to the auxin response; then, we examined the mode of action of a small-molecule of the piperazine type (designated ASP).

ASP is a piperazine compound with structural similarity to auxins; particularly, to PAA and 2,4-D. All three of these compounds have a benzene ring structure and a carboxyl group (or derivative), which are two common features recognized as critical for auxin activity; however, they differ with respect to the types and sites of the side chains on the benzene ring. In addition, cyclodipeptides and their derivative diketopiperazines (DKPs) synthesized by Pseudomonas aeruginosa also possess a 


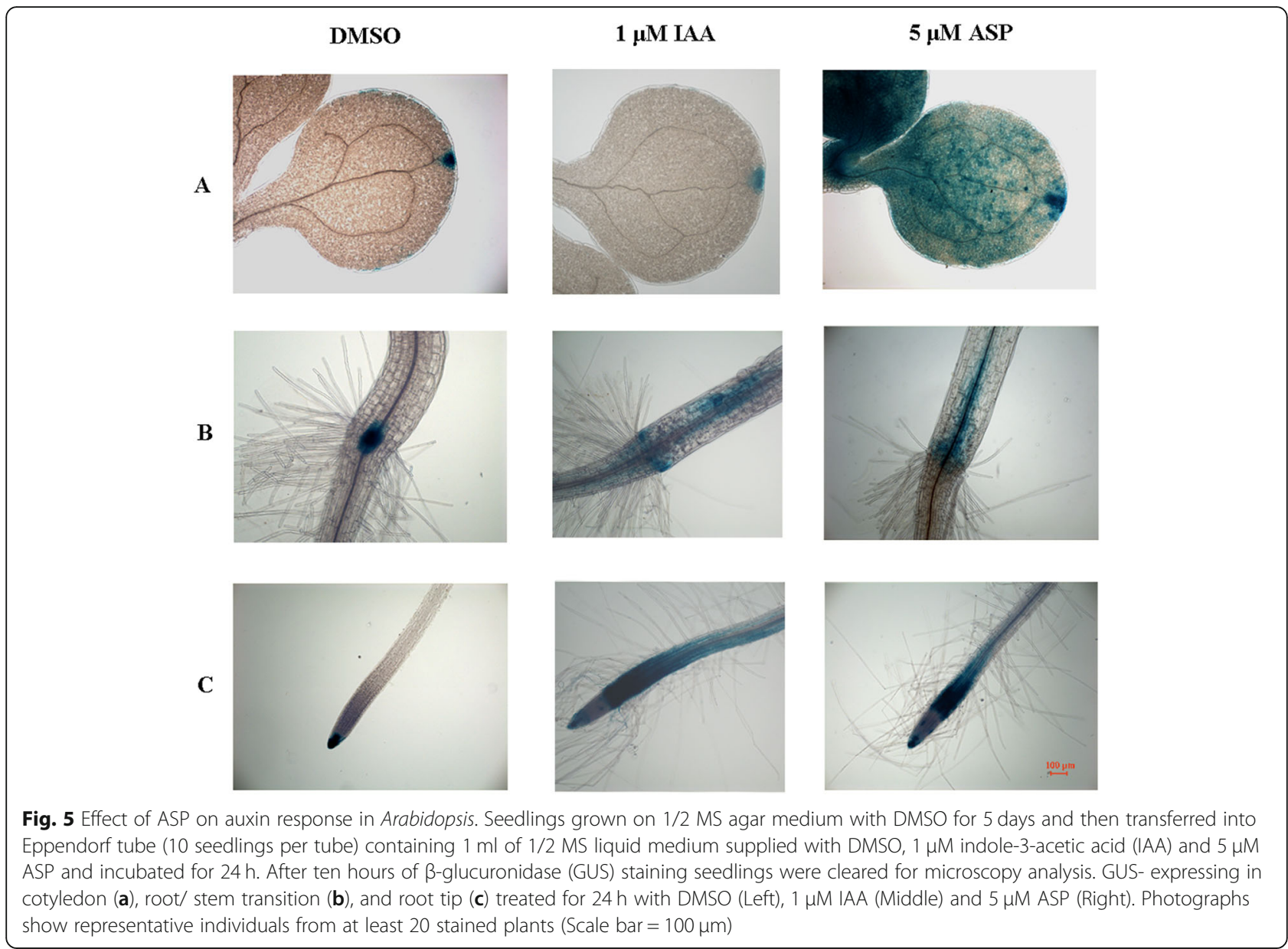

heterocyclic system and exhibit auxin-like activity [27]. Both ASP and DKPs belong among piperazines and show similar effects on modulating root architecture and inducing auxin response-related gene expression in Arabidopsis, which suggested ASP might be involved in the interaction of signaling-pathways between plants and bacteria.

In the experiments reported herein, we found that ASP effectively promoted hypocotyl elongation, whereas traditional auxins (e.g., IAA, 2,4-D, and NAA) had no effects in this respect under normal light conditions [28]. However, auxin overexpressing mutants, such as supperroot1 (sur1) and sur2, were characterized by a long hypocotyl and short roots, a phenotype that can be mimicked by exogenous application of the auxin analog picloram [29]. Nonetheless, it has been shown that the activity of picloram differs from that of 2,4-D with respect to the auxin receptor family member AFB5 [30]. Furthermore, yucca mutants were identified with long hypocotyls by activation tagging [31]. In addition to long hypocotyls, yucca plants showed phenotypes characteristic of elevated auxin levels during all stages of development; namely, yucca seedlings showed epinastic cotyledons and elongated petioles when grown under white light, similar to known auxin overproducing mutants sur 1 and sur 2 . In addition, altered conditions of nutrition, light, and temperature, can potentially contribute to the induction of hypocotyl elongation [32]. The findings of a study on auxin analogs revealed that certain new auxin analogs, acting as "pro-auxins," can diffuse efficiently to the hypocotyls, wherein they undergone cleavage at differing rates, thereby releasing functional auxins [22]. On the basis of the structure of ASP, we propose that it might also be hydrolyzed to yield an auxin-like active substance which, through tissuespecific localization, gains access to the hypocotyl-previously deemed inaccessible to exogenous auxins. The importance of such possibility warrants further study.

Given that auxins have been shown to inhibit primary root elongation while increasing lateral root development [33], we compared the effect of ASP and 2,4-D on primary root and lateral root growth, gravitropism, and root hair development. We found that primary root growth and gravitropism of WT seedlings was inhibited by ASP; similar to the phenotype induced by treatment with synthetic auxins. Furthermore, ASP induced a root morphology similar to the phenotype of MED18 loss-of- 


\section{A}

IAA2

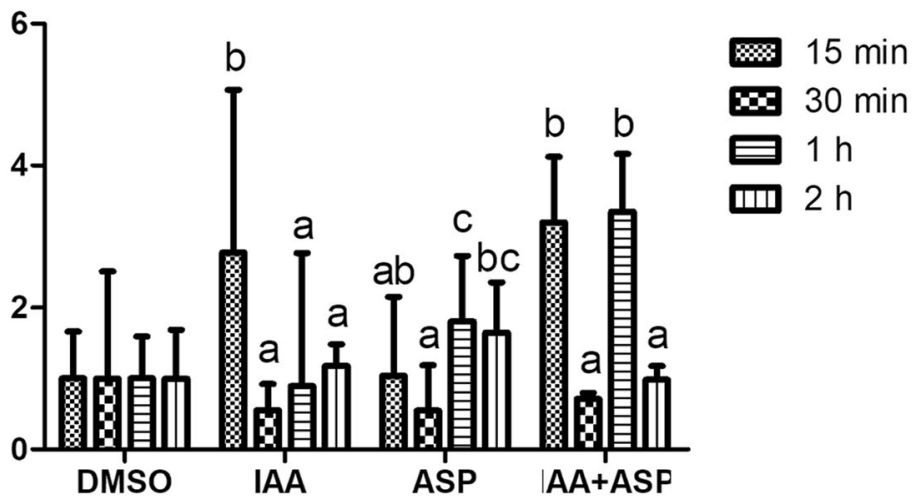

B

GH3.5

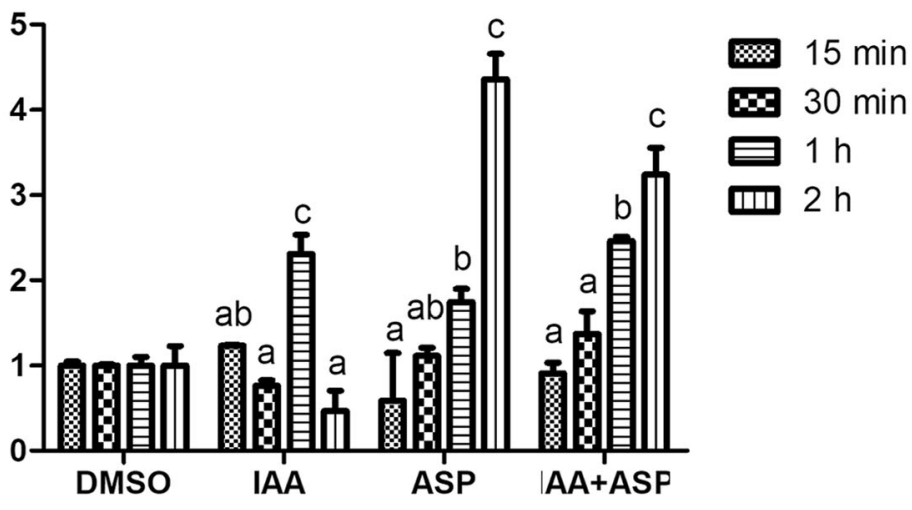

C

SAUR 23

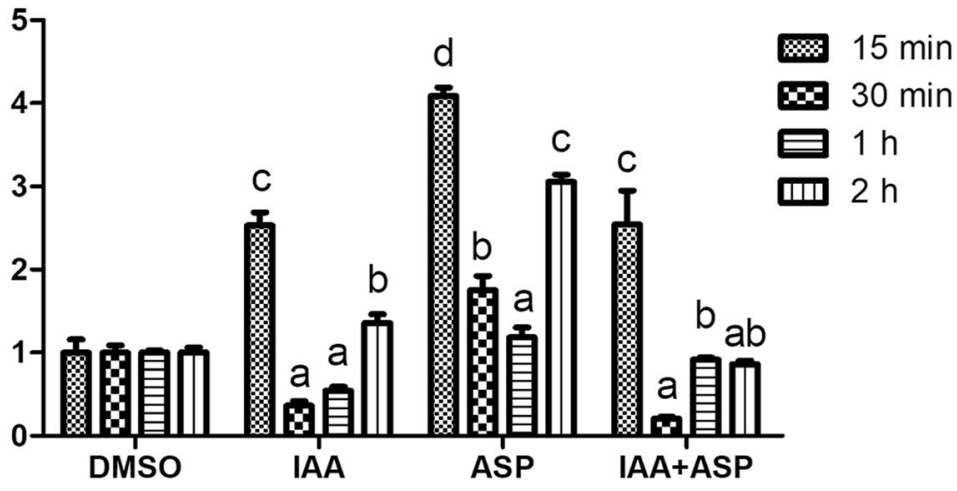

Fig. 6 RT-qPCR expression analyses of early auxin response genes. Six-day-old seedlings were treated with $5 \mu M$ ASP and $5 \mu M$ IAA for $0.5-2 \mathrm{~h}$. Expression level shown are the Means \pm SD from three biological replicates for each. Different letters indicate significant differences at $P<0.05$

function mutant. MED18 is involved in root morphogenesis and is important for cell viability. Mutant med18 exhibited slow primary root growth and increased root hair formation, and DNA damage occurred in dead cells of root meristems [34]. Meanwhile, ERF115 (Ethylene Response Factor) is helpful for sustaining meristematic activity via the enhancement of cell replenishment in response to cell damage and ERF115 expression is induced following cell damage. However, when we tested the expression levels of MED18 and ERF115 in the root tips of 6-day-old WT seedlings treated with 2 and $5 \mu \mathrm{M}$ ASP using RT-qPCR, the expression of these genes was 
distinct from that in the med18 mutant. A high expression level of MED18 and a reduced expression level of ERF115 resulted from $5 \mu \mathrm{M}$ ASP treatment (Additional file 9: Fig. S9). We accordingly deduced that the root morphological changes induced by ASP were not due to DNA damage or cell death. We confirmed this conclusion using PI staining: the detection of cell viability in the root tip meristem did not show any change under different concentration of ASP, $5 \mu \mathrm{M}$ IAA, or $30 \mathrm{nM} 2,4-$ D. Further, we observed that ASP had diverse effects on hypocotyl and root growth in auxin-signaling mutants. The hypocotyl length in tir1-1 and aux1-7 increased with 1-5 $\mu \mathrm{M}$ ASP relative to controls. However, the inhibitory effect on root growth had not been observed in these mutants. Accordingly, we suspected that ASP affected root and hypocotyl growth via different pathways.

On the basis of its structure and induced phenotypic characteristics in treated plants, we propose that ASP might be useful as a novel auxin analog. Histochemical staining using DR5::GUS confirmed that ASP interferes with the auxin response pathway, and RT-qPCR analysis indicated that early auxin response genes, including those in the AUX/IAA, GH3 and SAUR gene families, were significantly up-regulated via rapid induction by ASP, thereby confirming the auxin-like nature of ASP.

\section{Conclusions}

In this study, we identified the piperazine smallmolecule compound ASP based on high-throughput chemical screening. We found that ASP affected the early development of $A$. thaliana, with significant effects on leaf shape and venation pattern. Additionally, ASP had an inhibitory effect on the growth of primary roots but was found to promote hypocotyl elongation and reduced gravitropism in A. thaliana seedlings. Moreover, using RT-qPCR, we found that ASP induced the expression of early auxin response genes. Collectively, our findings indicate that ASP can regulate the growth and development of $A$. thaliana by activating an auxin response and inducing the expression of genes involved in the auxin signaling pathway. In addition to demonstrating the effects of the small-molecule compound ASP on A. thaliana seedlings at the phenotypic and cellular levels, our studies clearly highlight the advantages of using a chemical genetics approach to dissect the intricacies of plant hormone signaling-pathways.

\section{Methods}

\section{Plant materials and reagents}

Arabidopsis Columbia-0 wild type (WT), auxin related mutants such as tir1-1 (CS3798), aux1-7 (CS3074) and axr2-1 (CS3077) seed lines, and DR5::GFP and DR5:: GUS transgenic lines were used for phenotype and mode of action study. These seed lines were preserved in our laboratory. Q0990 line was used to screen chemicals. This line was a kind gift from Prof. Enrico Scarpella, University of Alberta, Canada. Seeds of A. thaliana were surface sterilized with 70\% ethanol and 10\% sodium hypochlorite and dried in sterile bench. The sterilized seeds were germinated on half-strength Murashige and skoog salt $(1 / 2 \mathrm{MS})$ medium supplemented with $0.1 \%$ sucrose and $0.7 \%$ plant agar, and grown at $22{ }^{\circ} \mathrm{C}-24^{\circ} \mathrm{C}$ with a light/dark cycle of $16 / 8 \mathrm{~h}$. All reagents were purchased from Sigma (St. Louis, MO, USA). Stock concentrations of $50 \mathrm{mM}$ ASP and $100 \mathrm{mM}$ 2,4-D were prepared in DMSO. The working concentrations were as follows: 10-100 nM 2,4-D, 0-30 $\mu \mathrm{M}$ ASP.

\section{Selection of $\mathbf{3 8 0 0}$ small-molecule compounds}

The Q0990 strain (provided by Enrico Scarpella of University of Alberta) contains the GFP protein and can excite green fluorescence in the vascular system. Screening process: Unbiased small molecule organic compounds were purchased from ChemBridge and stored in 96-well plates, one compound per well. Add one seed per well in a 96-well plate, and then add liquid 1/2 MS broth for a final concentration of $10 \mu \mathrm{M}$ compound and $0.1 \%$ DMSO. The same compound was treated simultaneously in two replicate plates with $1 / 2 \mathrm{MS}+\mathrm{DMSO}$ served as the control. The first pair of true leaves was observed after cold stratification at $4{ }^{\circ} \mathrm{C}$ in darkness for 2 days and light culturing at $23^{\circ} \mathrm{C}(16 \mathrm{~h}$ light $/ 8 \mathrm{~h}$ dark $)$ for 9 days. These were subsequently analyzed by confocal microscopy (Zeiss live 5).

\section{Phenotype investigation}

The seedlings grew in the chemical medium and the control group was photographed using a Nikon camera. The hypocotyls and root length of each $A$. thaliana seedling were measured using Image J software. At least twenty plants each from the control and treatment groups were analyzed.

\section{Microscopy observation of hypocotyl cell}

The hypocotyls of $A$. thaliana seedlings that had been cultured for 7 days were soaked in immobilized solution and fixed at $4{ }^{\circ} \mathrm{C}$ for more than $24 \mathrm{~h}$. Dehydration was carried out at an alcohol gradient of 30 to $50 \%$ to 70 to $75 \%$ to $80 \%$ to about $85 \%$ to about $90 \%$ to about $100 \%$. The embedding agent was then infiltrated into the material and polymerized at $4{ }^{\circ} \mathrm{C}$ for $24 \mathrm{~h}$. The sections were sliced at a thickness of $5 \mathrm{~mm}$ and stained to observe the cells under microscopy.

Growth, curvature, and analysis of Gravitropic sensitivity Wild-type A. thaliana seeds were cultured on $1 / 2 \mathrm{MS}$ medium for 4 days. Uniform seedlings were then selected and vertically grown in $2 \mu \mathrm{M}$-ASP medium for 24 
h. The plates were rotated clockwise $90^{\circ}$ to make the root parallel to the ground. We observed and photographed the root every $12 \mathrm{~h}$. Image J software was used to measure the curvature of 20 plants each in the control and treatment groups.

\section{Histochemical analysis}

Five-day-old Arabidopsis seedlings grown on MS medium with or without $5 \mu \mathrm{M}$ ASP were incubated at $37^{\circ} \mathrm{C}$ in a GUS staining solution (O'BioLab Co Ltd., Beijing, China) for $10 \mathrm{~h}$. Chlorophyll was removed by washing plants several times with $70 \%(\mathrm{v} / \mathrm{v})$ ethanol, and then observed under stereoscopic microscope zeiss, Axio Zoom.V16, Germany.

\section{RNA isolation and quantitative Reverese-transcription polymerase chain reaction}

Total RNA was isolated using TRIzol reagent (Invitrogen) according to manufacturer's instructions. Firststrand cDNA was synthesized with $5 x$ PrimeScript RT Master Mix (Takara). RT-qPCR was performed in the ABI (Quant studio 6, USA) using the $2 \times$ SYBT Premix Ex TaqTM (Takara) and analysed using "Quant studioReal-Time PCR" software. Primers are described in the Additional file 10: Table S1.

\section{Propidium iodide staining}

For fluorescence staining with PI, plants were transferred from the growth medium to $10 \mathrm{mg} / \mathrm{mL}$ PI solution for $2 \mathrm{~min}$. Seedlings were rinsed in phosphatebuffered saline (PBS) and mounted in water on microscope slides. Samples were observed under a laserscanning confocal microscope (LSCM: Zeiss 780; Germany) at wavelengths specific to PI fluorescence using a 561-nm excitation line [34].

\section{Statistical analysis}

For all experiments the overall data were statistically analyses in the spss19.0 software. All results were presented as the mean \pm SD. using GraphPad Prism 5.00 (GraphPad Software, San Diego, CA, USA) and analyzed using Student's t test or ANOVA (analysis of variance). Univariate analyses with a Tukey's-b (k)/Duncan (D) post hoc test were used for testing differences. Different letters are used to indicate means that differ significantly $(P<0.05)$.

\section{Supplementary Information}

The online version contains supplementary material available at https://doi. org/10.1186/s12864-020-07203-8.

Additional file 1: Fig. S1 Representative images of Arabidopsis WT (Col-0) seedlings grown on petri plates with medium supplemented with
2.4-D or ASP. DMSO was used as control. Concentrations are indicated under each image (Scale bar $=15 \mathrm{~mm}$ ).

Additional file 2: Fig. S2 Representative images of Arabidopsis WT (Col0) seedlings supplemented with 2,4-D or ASP. Seedlings were grown on $1 / 2$ MS liquid medium for nine days supplied with different concentrations of ASP or 2,4-D. DMSO was used as control. Concentrations are indicated under each image (Scale bar $=15 \mathrm{~mm}$ ).

Additional file 3: Fig. S3 Testing gravitropism of rotated roots with $2 \mu \mathrm{M}$ ASP treatment for $12-72 \mathrm{~h}$.

Additional file 4: Fig. $\mathbf{S 4}$ Representative images showing the root-hair phenotypes of Arabidopsis WT (Col-0) seedlings. Seedlings were grown on ASP or 2,4-D-supplemented medium for 6 days. DMSO was used as control. Concentrations are indicated on each image (Scale bar $=$ $500 \mu \mathrm{m})$.

Additional file 5: Fig. S5 Images of propidium iodide (PI)-stained root tip cells. Primary root tips from Col-0 seedlings, which grown on 1/2 MS medium with 0-5 MM ASP, $5 \mu \mathrm{M}$ IAA and $30 \mathrm{nM}$ 2,4-D for five days, stained with $10 \mathrm{mg} / \mathrm{ml} \mathrm{PI}$ for $2 \mathrm{~min}$. Images were acquired using a Zeiss LSCM 780 confocal microscope (Scale bar $=50 \mu \mathrm{m}$ ).

Additional file 6: Fig. $\mathbf{S 6}$ ASP promoted hypocotyl cell elongation. Semi-thin longitudinal section cut from hypocotyl tissue cells and observed with light microscopy (Scale bar $=50 \mu \mathrm{m}$ ).

Additional file 7: Fig. $\mathbf{S 7}$ Comparison of auxin signaling mutants (tir11, axr2-1, aux 1-7) with wild-type seedlings on hypocotyl and root growth. The seedlings were grown on medium supplemented with 0$5 \mu \mathrm{M}$ ASP for six days. A: Hypocotyl length. B: Root length. Means \pm SD values were shown, $N=30$ seedlings. C: Root hair number. D: Root hair length. Means \pm SD values were shown, $N=10$ seedlings. Micromolar concentrations are indicated on $\mathrm{X}$ axis.

Additional file 8: Fig. S8: Representative root hair phenotypes of auxinrelated mutants. A: Wild-type and mutant (tir 1-1, axr2-1, aux1-7) seedlings were grown on $1 / 2$ MS liquid medium for 6 days. B: Wild-type and mutants were treated with $4 \mu \mathrm{M}$ ASP for 6 days (Scale bar $=500 \mu \mathrm{m}$ ).

Additional file 9: Fig. S9: RT-qPCR expression analyses of ERF115 and MED18 to test the root meristem cell viability. The seedlings were grown on 1/2 MS medium supplemented with 2 and $5 \mu \mathrm{M}$ ASP for six days. DMSO was used as control.

Additional file 10: Table S1 Sequences of primers used in RT-qPCR.

\section{Abbreviations}

2,4-D: 2,4-Dichlorophenoxyacetic acid; 4-CI-IAA: 4-Chloroindole-3-acetic acid; A. thaliana: Arabidopsis thaliana; ABA: Abscisic acid; ACC : 1-Aminocyclopropane-1-carboxylic acid; ARF: Auxin response factor; ASP : 1-[(4Bromophenoxy) acetyl]-4-[(4-fluorophenyl) sulfonyl] piperazine; Aux/ IAA: Auxin/Indole-3-acetic acid; DMSO: Dimethyl sulfoxide; HSD: Honestly significant difference; IAA: Indoleacetic acid; IBA: Indole-3-butanoic acid; JA: Jasmonic acid; NAA: 1-Naphthylacetic acid; PAA: Phenylacetic acid; SA: Salicylic acid; SKP1: Suppressor of kinetochore protein 1; WT: Wide type; PI: Propidium iodide; PBS: Phosphate-buffered saline; LSCM: Laser scanning confocal microscope

\section{Acknowledgements}

We thank to Dr. Michael Deyholos of UBCO Biology for his helpful discussion and help with amending the article. We also thank to Enrico Scarpella of University of Alberta for his providing the Q0990 A. thaliana seeds and guidance in chemical screening. We would also like to thank Elizabeth Tokarz at the Yale University for her assistance with English language and grammatical editing.

\section{Authors' contributions}

XFY performed transcriptional level analysis and interpreted the experimental data. She was also a major contributor in writing the manuscript. XSQ performed propidium iodide (PI)-stained analysis and helped to revise the manuscript. XFY and XSQ are co-first authors; they contributed equally to the work. DLM performed experimental treatment and RT-qPCR analysis, and helped to draft the manuscript. ZSF participated in the chemical characterization and phenotype identification. LYX did the cytological 
observation. ZX designed the study and provided the guidance of the whole research work. All authors read and approved the final manuscript before submission.

\section{Funding}

This work was supported by National Nature Science Foundation of China (30900771) and Scientific Research Program of Beijing Municipal Education Commission (KM201510028011). The funders had no role in study design, data collection and analysis, decision to publish to publish, or preparation of the manuscript.

\section{Availability of data and materials}

All data generated or analysed during this study are included in this published article and its supplementary information files.

IAA2 (AT3G23030), GH3.5 (AT4G27260), SAUR23 (AT5G18060), PIN2

(AT5G57090), ERF115 (AT5G07310), MED18 (AT2G22370).

\section{Ethics approval and consent to participate}

Not applicable.

\section{Consent for publication}

Not applicable.

\section{Competing interests}

The authors declare that they have no competing interests.

Received: 17 March 2020 Accepted: 28 October 2020

Published online: 11 November 2020

\section{References}

1. Enders TA, Strader LC. Auxin activity: past, present, and future. Am J Bot. 2015;102(2):180-96.

2. Paque S, Weijers D. Q\&a: Auxin: the plant molecule that influences almost anything. BMC Biol. 2016;14(1):67.

3. Quint M, Gray WM. Auxin signaling. Curr Opin Plant Biol. 2006;9(5):448-53.

4. Weijers DWD. Transcriptional responses to the auxin hormone. Annu Rev Plant Biol. 2016:67(1):539-74.

5. Rock CD, Sun X. Crosstalk between $A B A$ and auxin signaling pathways in roots of Arabidopsis thaliana (L.) Heynh. Planta. 2005;222(1):98-106.

6. Chandler JW. Auxin as compere in plant hormone crosstalk. Planta. 2009; 231(1):1-12.

7. Duran-Medina Y, Diaz-Ramirez D, Marsch-Martinez N. Cytokinins on the move. Front Plant Sci. 2017:8:146.

8. Yip WK, Yang SF. Effect of thidiazuron, a cytokinin-active urea derivative, in cytokinin-dependent ethylene production systems. Plant Physiol. 1986;80(2): 515-9.

9. Mendez-Bravo A, Francisco Ruiz-Herrera L, Cruz-Ramirez A, Guzman P, Martinez-Trujillo M, Ortiz-Castro R, Lopez-Bucio J. CONSTITUTIVE TRIPLE RESPONSE1 and PIN2 act in a coordinate manner to support the indeterminate root growth and meristem cell proliferating activity in Arabidopsis seedlings. Plant Sci. 2019;280:175-86.

10. Beyer EM, Morgan PW. Abscission: the role of ethylene modification of auxin transport. Plant Physiol. 1971;48(2):208-12.

11. She XP, Song XG. Cytokinin- and auxin-induced stomatal opening is related to the change of nitric oxide levels in guard cells in broad bean. Physiol Plant. 2006;128(3):569-79.

12. Grabov A, Blatt MR. Membrane voltage initiates $\mathrm{Ca}^{2+}$ waves and potentiates $\mathrm{Ca}^{2+}$ increases with abscisic acid in stomatal guard cells. Plant Biol. 1998;95: 4778-478.

13. Zhao Y, Chow TF, Puckrin RS, Alfred SE, Korir AK, Larive CK, Cutler SR. Chemical genetic interrogation of natural variation uncovers a molecule that is glycoactivated. Nat Chem Biol. 2007;3(11):716-21.

14. Van de Wouwer D, Vanholme R, Decou R, Goeminne G, Audenaert D, Nguyen L, Hofer R, Pesquet E, Vanholme B, Boerjan W. Chemical genetics uncovers novel inhibitors of lignification, including $p$-iodobenzoic acid targeting CINNAMATE-4-HYDROXYLASE. Plant Physiol. 2016;172(1):198-220.

15. Tsuchiya Y, Vidaurre D, Toh S, Hanada A, Nambara E, Kamiya Y, Yamaguchi S, McCourt P. A small-molecule screen identifies new functions for the plant hormone strigolactone. Nat Chem Biol. 2010;6(10):741-9.

16. Sim DS, Flaumenhaft R. The platelet as a model for chemical genetics. Chem Biol. 2003;10(6):481-6.
17. Shirakawa M, Ueda H, Shimada T, Hara-Nishimura I. Myrosin cells are differentiated directly from ground meristem cells and are developmentally independent of the vasculature in Arabidopsis leaves. Plant Signal Behav. 2016;11(4):e1150403.

18. Qin H, Huang R. Auxin controlled by ethylene steers root development. Int J Mol Sci. 2018;19(11):3656.

19. Ge L, Chen R. Negative gravitropism in plant roots. Nature Plants. 2016;2(11): 16155.

20. Dolan L. The role of ethylene in root hair growth in Arabidopsis. J Plant Nutr Soil Sci. 2001;164(2):141-5.

21. Achard P, Renou JP, Berthome' R, Harberd NP, Genschik P. Plant DELLAs restrain growth and promote survival of adversity by reducing the levels of reactive oxygen species. Curr Biol. 2008;18(9):656-60.

22. Savaldi-Goldstein S, Baiga TJ, Pojer F, Dabi T, Butterfield C, Parry G, Santner A, Dharmasiri N, Tao Y, Estelle M, et al. New auxin analogs with growthpromoting effects in intact plants reveal a chemical strategy to improve hormone delivery. Proc Natl Acad Sci U S A. 2008;105(39):15190-5.

23. Schellmann S, Schnittger A, Kirik V, Wada T, Okada K, Beermann A, Thumfahrt J, Jurgens G, Hulskamp M. TRIPTYCHON and CAPRICE mediate lateral inhibition during trichome and root hair patterning in Arabidopsis. EMBO J. 2002;21(19):5036-46.

24. Woodward AW, Bartel B. Auxin: regulation, action, and interaction. Ann Bot. 2005;95(5):707-35.

25. Mockaitis K, Estelle M. Auxin receptors and plant development: a new signaling paradigm. Annu Rev Cell Dev Biol. 2008;24(1):55-80.

26. $\mathrm{Ma} \mathrm{Q}, \mathrm{Grones} \mathrm{P}$, Robert $\mathrm{S}$. Auxin signaling: a big question to be addressed by small molecules. J Exp Bot. 2018;69(2):313-28.

27. Ortiz-Castro R, Diaz-Perez C, Martinez-Trujillo M, del Rio RE, Campos-Garcia J, Lopez-Bucio J. Transkingdom signaling based on bacterial cyclodipeptides with auxin activity in plants. Proc Natl Acad Sci U S A. 2011;108(17):7253-8.

28. Song $Y$. Insight into the mode of action of 2,4-Dichlorophenoxyacetic acid (2,4-D) as an herbicide. J Integr Plant Biol. 2014;56(2):106-13.

29. Boerjan W, Cervera MT, Delarue M, Beeckman T, Dewitte W, Bellini C, Caboche C, Van Onckelen H. Van Montagu M, Inzé D: Superroot, a recessive mutation in Arabidopsis, confers auxin overproduction. Plant Cell. 1995;7(9): 1405-19.

30. Walsh TA, Neal R, Merlo AO, Honma M, Hicks GR, Wolff K, Matsumura W, Davies JP. Mutations in an auxin receptor homolog AFB5 and in SGT1b confer resistance to synthetic picolinate auxins and not to 2,4Dichlorophenoxyacetic acid or indole-3-acetic acid in Arabidopsis. Plant Physiol. 2006;142(2):542-52.

31. Weigel D, Ahn JH, Bla'zquez MA, et al. Activation tagging in Arabidopsis. Plant Physiol. 2000;122(4):1003-13.

32. Gray WM, Ostin A, Sandberg G, Romano CP, Estelle M. High temperature promotes auxin-mediated hypocotyl elongation in Arabidopsis. Proc Natl Acad Sci U S A. 1998;95(12):7197-202.

33. Ulmasov T, Liu ZB, Hagen G, Guilfoyle TJ. Composite structure of auxin response elements. Plant Cell. 1995;7(10):1611-23.

34. Raya-Gonzalez J, Oropeza-Aburto A, Lopez-Bucio JS, Guevara-Garcia AA, de Veylder L, Lopez-Bucio J, Herrera-Estrella L. MEDIATOR18 influences Arabidopsis root architecture, represses auxin signaling and is a critical factor for cell viability in root meristems. Plant J. 2018;96(5):895-909.

\section{Publisher's Note}

Springer Nature remains neutral with regard to jurisdictional claims in published maps and institutional affiliations.

Ready to submit your research? Choose BMC and benefit from:

- fast, convenient online submission

- thorough peer review by experienced researchers in your field

- rapid publication on acceptance

- support for research data, including large and complex data types

- gold Open Access which fosters wider collaboration and increased citations

- maximum visibility for your research: over $100 \mathrm{M}$ website views per year

At $\mathrm{BMC}$, research is always in progress.

Learn more biomedcentral.com/submissions 